\title{
The Critical Role of Imaging in Primary Progressive Multiple Sclerosis
}

\author{
Yuyun Yueniwati $^{1 *}$, Dotty Inggrianita ${ }^{2}$ \\ ${ }^{1}$ Radiology Department, Faculty of Medicine, Universitas Brawijaya, Malang, Indonesia; ${ }^{2}$ Neurology Department, Faculty of \\ Medicine, Universitas Brawijaya, Malang, Indonesia
}

Edited by: Ksenija Bogoeva-Kostovska Citation: Yueniwati Y, Inggrianita D. The Critical Role of maging in Primary Progressive Multiple Sclerosis. Open
Access Maced J Med Sci. 2020 Oct 15; 8(C): 195-200 Access Maced J Med Sci. 2020 Oct 15; 8(C): $195-200$ Multiple sclerosis; Primary progressive type; Keywords: Multiple sclerosis; Primary progressive type;
Imaging
*Correspondence: Yuyun Yueniwati, Radiology *Correspondence: Yuyun Yueniwati, Radiology
"Contment, Faculty of Medicine, Universitas Brawijaya, Malang, Indonesia. E-mail: yuyun@ub.ac.id Received: 11-Apr-2020 Revised: 01-Oct-2020 Copyright: $\odot 2020$ Yuyun Yueniwati, Dotty Inggrianita
Funding: This research did not receive any financial (n) any financia Competing Interests: The authors have declared that no competing interests exist Open Access: This is an open-access article distributed under the terms of the Creative Commons Attribution-
NonCommercial 4.0 International License (CC BY-NC 4.0)

\begin{abstract}
BACKGROUND: Multiple sclerosis (MS) is an autoimmune-related neurological disorder as a result of chronic inflammation and demyelination which affect the central nervous system. The number of MS cases in Indonesia is about 1-5/100.000 people. This disease causes disability and requires immense treatment costs. One of the MS subtypes is primary progressive which is marked by the decline of neurological functions since the disease onset without any period of relapse attacks.

CASE REPORT: A 59-year-old female came with complaints on spinning dizziness, accompanied by vomiting and waddling for the past month before hospital administration. Based on physical examination, we found multiple crania nerve palsies on the cranial nerve III, IV, V, VI, and VII on the left side. Initially, it suspected as an intracranial lesion in the cerebellopontine angle. However, brain computed tomography scan showed a normal result. Brain magnetic resonance imaging (MRI) was conducted and we found multiple lesions on the supratentorial and infratentoria region. The second patient was a 24-year-old female complained about waddling gait for the past year, which grew heavier. Based on physical examination, we found a cerebellar sign on the left side, cerebellar ataxia, paraparesis of lower extremity, and paresthesia. Brain MRI with contrast displayed white matter periventricular and infratentoria lesion.
\end{abstract}

CONCLUSION: In both cases, radiological imaging examination, which was MRI with contrast, was beneficial in the establishment of patient's diagnosis, which made therapeutic management suitable as needed.

\section{Introduction}

Multiple sclerosis (MS) is a neurological disorder caused by chronic autoimmune demyelination related to the central nervous system (CNS) [1], [2]. Pathologies of demyelination disease are (1) destruction of the myelin sheath on nerve fibers, which the other nervous tissue elements such as axons, neuron cells, and their supporting structures remain intact, which is proven by the absence of Wallerian degeneration or other secondary degeneration; (2) infiltration from inflammatory cells to the perivascular region; and distribution from lesion to the white matter, either separate multiple small lesion focus or large lesions scattered from one or more lesions [3]. MS affects approximately 400,000 people in the United States or about $140 / 100,000$ and in Europe is around $108 / 100,000$, which are mostly found on young individuals at the age of 20-50 [4], [5]. The prevalence of MS in Indonesia ranges at an estimate of $0-5 / 100,000$ [6]. The prevalence of MS in Indonesia ranges at an estimate of $5 / 100.000$ and among $85 \%$ of MS patients suffer relapse and remission phase [6]. MS is thought to be the result of complex interactions between genetics and environment [2], [7]. Environment factors which play a role, among which are exposure to infections, exposure to sunlight, and Vitamin D. Pathogens are suspected to be involved on the MS pathogenesis are human herpesvirus type 6, Epstein-Barr virus, and Mycoplasma pneumonia [8]. Manifestations of MS can vary greatly. The quite typical symptoms consist of fatigue, depression, cognitive dysfunction, soreness, defecation and urination disturbance, visual impairment, vertigo, ataxia, tremors, extrapyramidal symptoms, sensory disturbance, and motor disorder [9].

Early MS diagnosis is primarily based on clinical features. McDonald criteria are the most common MS diagnosis criteria of The International Panel on Diagnosis of MS [10].

There are four clinical forms of MS, which are relapsing-remitting MS (RRMS), secondary progressive MS (SPMS), primary progressive MS (PPMS), and progressive relapsing MS (PRMS) [4].

Epidemiologically, airway infection can be related to MS relapse. Therefore, pathogen infection is believed to trigger autoimmune response toward CNS myelin on individuals who are genetically vulnerable through molecular mimicry of infection agents with myelin in the CNS, which leads to protein self-destruction as well as inflammation and demyelination disease [8].

Magnetic resonance imaging (MRI) is crucial to diagnose and manage MS. MS lesions are often found 
on the periventricular, juxtacortical, infratentorial region of the brain, and spinal cord [1], [5].

Therapies for MS are classified into two types, which are immunomodulation therapy for the underlying immune process and therapy to reduce disease symptoms. In addition, non-pharmacological therapy in the form of physiotherapy and occupational therapy are also given [5], [9].

\section{Case Report}

The first case was the 59-year-old male who came to the emergency department with complaints on spinning dizziness and waddling gait for the last six weeks which became heavier accompanied by vomiting. Two months before hospital administration, the patient complained that he had difficulty in swallowing, thickening of the left side of his face, and closing of the left eyelid. The patient had a cardiac arrest when he was at the Emergency Department of Saiful Anwar Hospital, Malang, Indonesia. There was no history of similar complaints before and no similar complaint was experienced by the family.

Based on physical examination, the patient was intubated with GCS E4 VX M6, blood pressure at $100 / 70 \mathrm{mmHg}$, heart rate at 110 beats/min, and respiratory rate at $20 \mathrm{times} / \mathrm{min}$. There were also multiple cranial nerves III, IV, V, VI, and VII palsies of the left side LMN type. Based on the EMG result, moderate axonal demyelinating motor and sensory polyneuropathy and bilateral phrenic nerve palsy were found. Blood gas analysis presented respiratory failure.

Brain computed tomography scan showed a normal result. Brain MRI result with contrast displayed (1) hyperintense lesion on left globus pallidus, mesencephalon, pons, and medulla oblongata suspected as MS with differential diagnosis vasculitis or metabolic disease, (2) chronic punctate hemorrhage with differential diagnosis cavernous malformation on the left temporal lobe, and (3) senile brain atrophy with Grade I Fazekas scale (Figure 1).

The patient was diagnosed as a suspect for PPMS. Throughout treatment, the patient received pulse methylprednisolone $4 \times 250 \mathrm{mg}$ for 5 days which was then

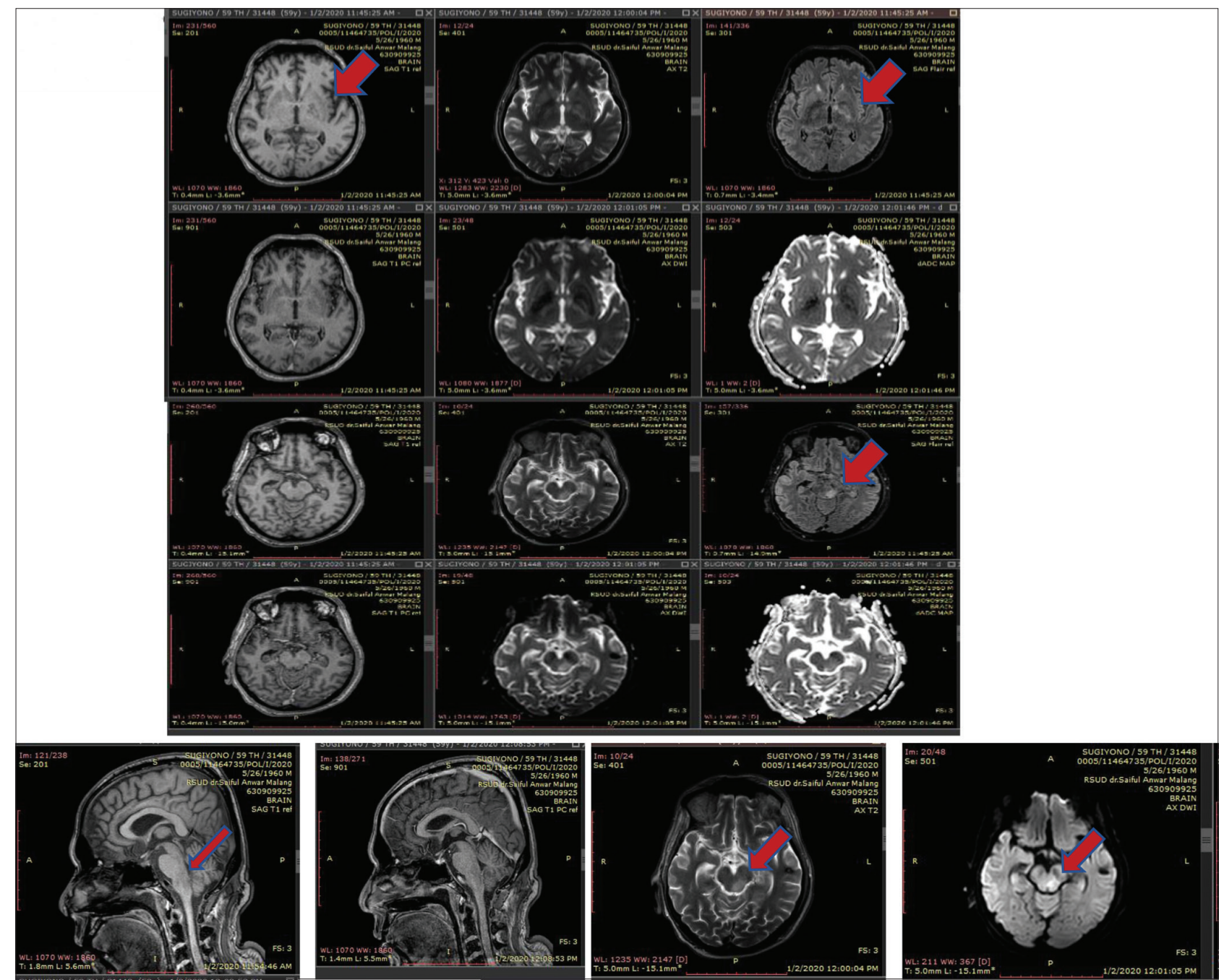

Figure 1: Brain MRI with contrast 
continued with prednisone oral at $1 \mathrm{mg} / \mathrm{kg}$ bodyweight that was reduced gradually. The patient experienced respiratory failure and was intubated and placed on a ventilator during treatment in ICU. Provisions of corticosteroid according to therapy guideline did not give meaningful recovery on the patient, so plasmapheresis was added into the therapy. Throughout the treatments in ICU using ventilator, the patient had suffered from sepsis due to pneumonia related to ventilator use, with the result of bacterial culture was sensitive to the antibiotic tigecycline.

The second case was a 24-year-old female, complained cerebellar ataxic gait for the past year, accompanied with a double vision which grew increasingly heavy, lower back pain which spread to both of her legs, thickness from the hip to the sole, and weakness on both legs. These complaints never got better. She had an epileptic seizure 1 month before admission.

On physical examination showed that the patient's GCS E4 V5 M6 with blood pressure at 110/70 $\mathrm{mmHg}$, heart rate at 82 beats/min, and respiratory rate at $18 \mathrm{times} / \mathrm{min}$. It was found that there was a left cranial nerve VII palsy LMN type. We found positive cerebellar signs, dysmetria on the left side, balance disorder indicated by a positive Romberg test. There was also paraparesis on the lower extremity with MMT Scale 4. Physiological reflex increased by +3 on bilateral BPR, TPR, KPR, and APR (Figure 2).

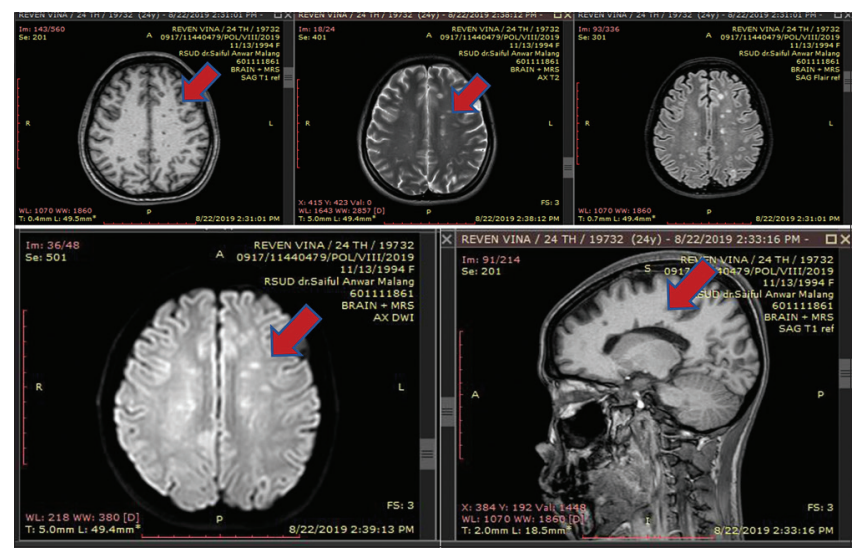

Figure 2: Brain MRI with contrast

Brain MRI result with contrast showed (1) multifocal white matter lesion on the right lateral ventricle, right and left frontotemporal lobe, cerebellum, mesencephalon, and pons due to MS and (2) left thalamus chronic infarction. EEG result showed mild diffuse encephalopathy. We performed a perspiration test; the result indicated autonomic dysfunction at the T6 vertebrae level. The patient was planned to undergo a cervicothoracic MRI with contrast.

Our patient was diagnosed as suspect PPMS. Throughout treatment, the patient received pulse methylprednisolone $4 \times 250 \mathrm{mg}$ for 5 days. Previously, the patient had routinely consumed antiepileptic drug phenytoin for $2 \times 100 \mathrm{mg}$ and was continued during treatment at the Saiful Anwar Hospital.

\section{Discussion}

MS is thought to be the result of complex interactions between genetics and environment. Genomic studies showed that immune system plays an important role as the central influence on the pathogenesis of MS through MHC class II which is mapped in HLADRB5*0101, HLA-DRB1*1501, HLA-DQA1*0102, and HLA-DQB1*0602 [3]. A study conducted on identical twins presented concordance ratio was $30 \%$. Fathers who suffer from MS have the risk to inherit the MS to their sons by $1 \%$ and their daughters by $2 \%$. Meanwhile, the siblings of an individual with MS have the risk of contracting MS by $3-4 \%$, as do non-identical siblings [2], [8].

The diagnosis of MS is established clinically and there is no definite examination for MS. Diagnosis establishment requires neurological assessment and clinical observation in a certain period of time [2], [5], [10].

In general, MS patients come with remission and exacerbation symptom and sign. Diagnosis for MS must be considered on patients who come with their first episode of neurological symptoms or signs which support a demyelination process and have no other possible causes. The first episode of this clinical manifestation is called Clinically Isolated Syndrome (CIS) [4].

Clinical manifestations which should be suspected as MS symptoms comprise of the decrease of visual acuity on either both of eyes which may be accompanied with soreness during eyeball movement, double vision, sensory disturbance and/or weakness, balance problems, and positive Lhermitte's sign [3] (Table 1).

Table 1: McDonald criteria 2010 [10]

\begin{tabular}{ll}
\hline Clinical presentation & Additional data needed for diagnosis \\
\hline$\geq 2$ clinical attacks and objective & None \\
evidence of $\geq 2$ lesions & \\
$\geq 2$ clinical attacks and objective & DIS: An additional attack implicating a different CNS \\
evidence of 1 lesion & site OR by MRI \\
1 clinical attack and objective & DIT: An additional clinical attack \\
evidence of $\geq 2$ lesions & OR by MRI \\
& OR \\
& CSF-specific oligoclonal bands \\
1 clinical attack and objective & DIS: An additional clinical attack implicating a different \\
evidence of 1 lesion & CNS site \\
& OR by MRI \\
& OR \\
& DIT: An additional clinical attack \\
& OR by MRI \\
& OR \\
& CSF-specific oligoclonal bands \\
\hline
\end{tabular}
Adapted from Thompson AJ, Banwell BL, Barkhof, et al. Diagnosis of multiple sclerosis: 2017 revisions of the McDonald criteria. Lancet Neurol. 2018; 17:162-73. CNS: Central nervous system, CSF: Cerebrospin fluid, DIS: Disseminated in space, DIT: Disseminated in time, MRI: Magnetic resonance imaging. ${ }^{2}$ DIS by
flus fluid, DIS: Disseminated in space, DIT: Disseminated in time, MRI: Magnetic resonance imaging. DIS by
MRI: New lesions on follow-up imaging or both gadolinium-enhancing and non-enhancing lesions on single MRI: New lesions on follow-up imaging or both gadolinium-enhancing and non-enhancing lesions on sing
MRI, ${ }^{D}$ DIS by MRI: $\geq 1$ symptomatic or asymptomatic lesion in $\geq 2$ areas including cortical/juxtacortical, MRI, ${ }^{b}$ DIS by MRI: $\geq 1$ symptomatic or a
periventricular, infratentorial, or spinal.

Natural immune response plays a positive role in the initiation and progressivity of MS disease. The link between pathogen-associated molecule and toll-like receptors will activate the dendritic cell (DC). Immature DC becomes semi-mature and induces regulatory $T$ cells to produce cytokine IL-10 and TGF- $\beta$. After DC has matured, DC induces $\mathrm{CD} 4^{+} \mathrm{T}$ cells to differentiate into Th1, Th2, and Th17. The differentiation of T cell into Th1 will lead to inflammation [3]. 
Adaptive immune response triggered by APC which presents specific antigen to $T$ lymphocytes, thus activating $\mathrm{CD}^{+}$and $\mathrm{CD}^{+} \mathrm{T}$ lymphocyte. Cells that may be involved as APC are B lymphocyte, DC, microglia, and macrophage. TH1 secretes pro-inflammatory cytokine interferon-gamma, while Th2 secretes antiinflammatory cytokine IL-4 and IL-13. Th17 secretes IL-17, IL-21, IL-22, and IL-26. Th17 also triggers the inflammation process on MS. During MS, Th1 and TH17 cells migrate to the CNS and cause demyelination and axonal lesions. $\mathrm{CD}^{+}$cells also kill glia cells which cause axons to be exposed. CD8 ${ }^{+} T$ cells attach to axon and increase vascular permeability and trigger the death of oligodendrocytes [1], [2], [8].

B cells play a role in the pathogenesis of MS by transforming into plasma cells and producing antibodies. The presence of a polyclonal antibody in the cerebrospinal fluid of MS patients is known as oligoclonal bands [9] (Figure 3).

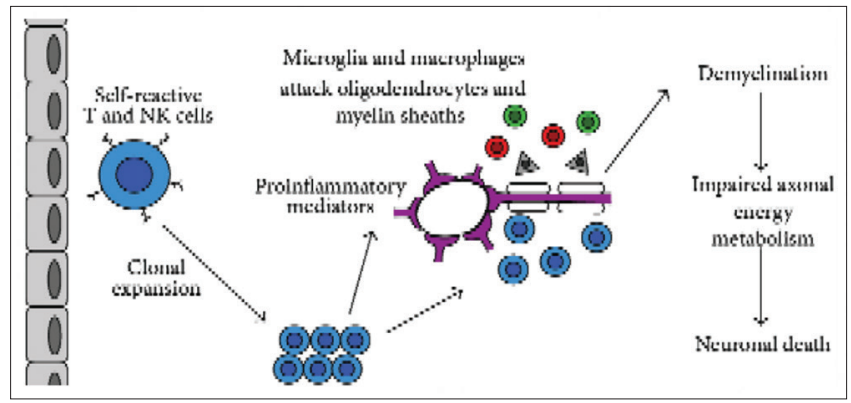

Figure 3: Immunology process on the central nervous system

There are four clinical types of MS, which are RRMS, SPMS, PPMS, and PRMS [2] RRMS is the most often occurring type, accounting for $85 \%$. RRMS is more frequently found on women than on men with a ratio of $2: 1$ and average onset at the age of 29. RRMS is marked by acute attacks (relapse) which are followed by full recovery (remission) and partial recovery that contrasts PPMS. The prevalence of PPMS is $10-15 \%$ of MS. On average, PPMS is diagnosed at the age of 40 and its prevalence for both men and women is the same. PPMS is marked by the continuation and irreversibility of disease progressivity [2] [11] (Figure 4).

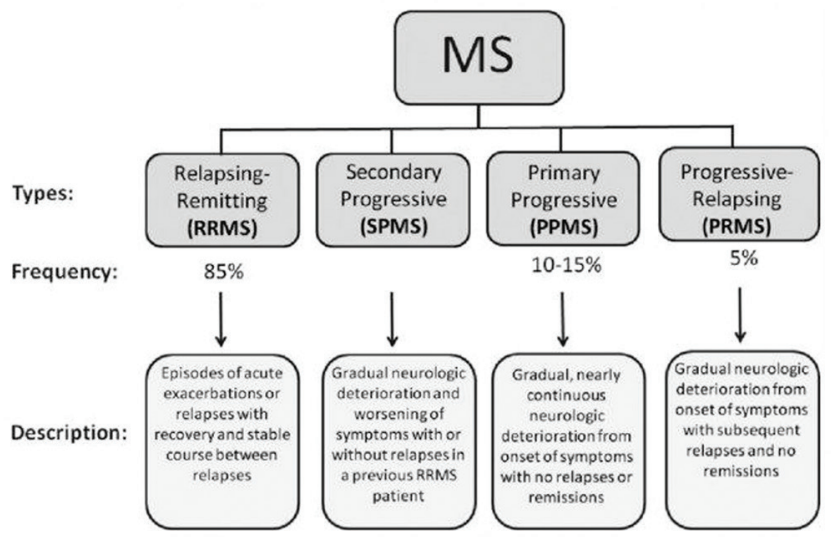

Figure 4: Multiple sclerosis subtypes [11]
MS can resemble other diseases, which means that before coming to the diagnosis of MS, other differential diagnoses, for instance, CNS infection, cerebrovascular disease, and other autoimmune diseases, such as NMO, SLE, neoplasm, and metabolic caused must be removed first [5].

Radiological imaging, especially MRI, is crucial for diagnosing and managing MS [5]. MRI can tell apart lipids from water. Myelin primarily consists of lipid, meaning that regions that experience demyelination contain more water. This in turn causes the demyelinated region to show darker colors on $\mathrm{T} 1$ and bright colors on T2. On T1, water is colored darker and lipid is colored brighter, and conversely, on T2 [5], [7].

MRI imaging of $\mathrm{T} 1$ is more sensitive when contrast is used, for instance, gadolinium diethylenetriaminepentaacetic acid. Gadoliniumenhanced can indicate the presence of disturbance at the blood-brain barrier on the acute lesion and the presence of a new lesion in CNS (particularly brain).

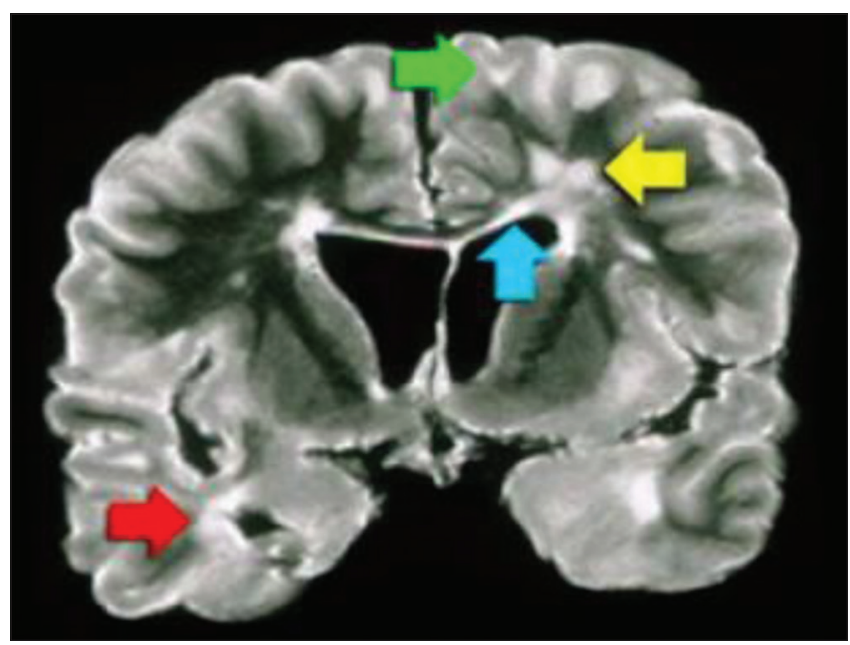

Figure 5: Specific location of MS [8]

Therefore, T1 MRI with contrast can distinguish acute and chronic lesions. MS lesions can often be found in the periventricular, juxtacortical, and infratentorial region of the brain and spinal cord. Dawson's finger is a specific lesion in MS [10]. What is meant by Dawson's finger is an ovoid lesion around the ventricle which exists due to demyelination along the small veins in cerebri [1], [8] (Figures 5 and 6).

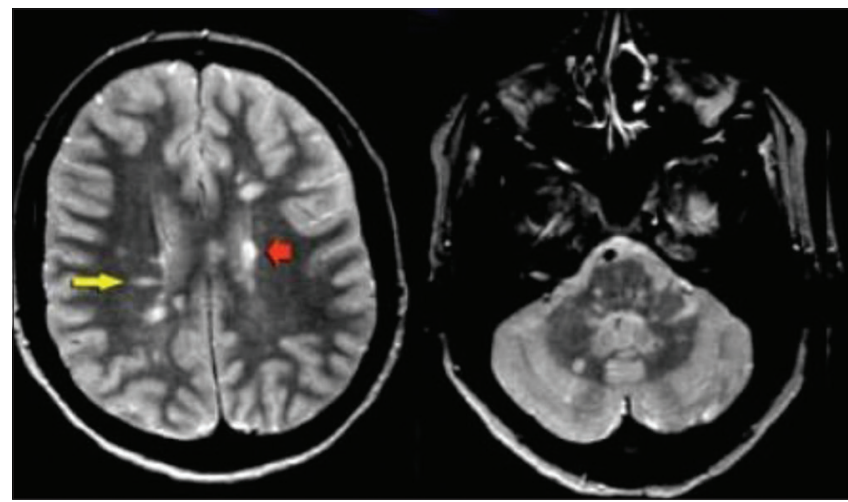

Figure 6: Image of Dawson's finger [8] 
Other supporting examination which can assist in establishing the diagnosis for MS is evoked potential and cerebrospinal fluid examination, though the two are not specific for MS. For EVP, if linear with lesion then it can be objective clinical evidence. For cerebrospinal fluid examination, IgG and oligoclonal band have high sensitivity, but they are not specific to MS [5].

Disease modification therapy is mainly conducted for controlling the underlying processes of disease instead of curing or even reversing the damages due to earlier attacks. Provided medications also aim to lessen disease symptoms. Therapies used for the acute phase are methyl prednisolone, among others. In the event that contraindication occurs for steroid administration, plasma exchange is given for severe attacks or when the steroid is proven ineffective [3].

The purpose of treatments on PPMS patients is to stabilize the disease, to provide supportive therapy, and to improve living quality. Until today, there has yet a recommended medication for PPMS [4].

Prognosis of MS patients presents $30 \%$ of which experience disabilities within 20-35 years. Only $5-10 \%$ of patients have a mild case of MS. Most individuals who have MS die due to secondary complications, such as pneumonia or kidney diseases, while only a small fraction die due to suicide or causes not relevant to MS. Relapsing and remitting can happen after 15 years [2].

For the first case, the patient experienced vertigo, spinning sensation, and multiple cranial nerve palsy which was not typical to MS, initially suspected as a form of intracranial lesion heading toward a tumor in the cerebellopontine angle.

For the second patient, the appearing symptoms were cerebellar ataxia, which is accompanied by weakness in her lower extremities, paresthesia at the T6 myelum level. On both patients, we found neither body heat nor infection signs which constantly for more than $24 \mathrm{~h}$. Supporting examination was done through brain MRI with contrast which led to the diagnosis of MS.

Clinically, both patients fulfilled the CIS criteria, which are monofocal or multifocal disorder and generally related to the optic nerve, cerebellum, spinal cord, and cerebral hemisphere, all of which were able to be used for McDonalds criteria revised in 2010 as a diagnosis for MS [10].

Based on anamnesis and physical and supporting examinations for these patients, diagnosis of MS favored toward primary progressive subtype.

The patients were given high doses corticosteroid therapy that is methylprednisolone for 5 days. Both first and second patients did not show good responses and complaints still remained. For the following management, patients were given immunosuppressant drugs such as azathioprine and methotrexate. The first patient suffered from secondary complications and required treatments in ICU with a ventilator. Meanwhile, the second patient was able to carry out therapy through the polyclinics, despite still having persistent complaints.

\section{Conclusion}

MS is a neurological disorder as a result of chronic autoimmune demyelination which is related to the CNS. The diagnosis for MS used McDonalds criteria revised in 2010. Therapies consisted of pharmacological, which comprised causative and symptomatic therapy, as well as non-pharmacological such as physiotherapy. In both cases, MRI, as a radiological imaging examination with contrast, was found to be very helpful in establishing a patient's diagnosis; therefore, therapeutic management can be given according to their conditions.

\section{References}

1. Filippi M, Rocca MA, Ciccarelli O, De Stefano N, Evangelou N, Kappos L, et al. MRI criteria for the diagnosis of multiple sclerosis: MAGNIMS consensus guidelines. LancetNeurol. 2016;15(3):292303. https://doi.org/10.1016/s1474-4422(15)00393-2 PMid:26822746

2. Sand IK. Classification, diagnosis, and differential diagnosis of multiple sclerosis. Curr Opin Neurol. 2015;28(3):193-205. https://doi.org/10.1097/wco.0000000000000206 PMid:25887774

3. Thompson AJ, Baranzini SE, Geurts J, Hemmer B, Ciccarelli O. Multiple sclerosis. Lancet. 2018;391(10130):1622-1636. https:// doi.org/10.1016/s0140-6736(18)30481-1

4. Correale J, Gaitán MI, Ysrraelit MC, Fiol MP. Progressive multiple sclerosis: From pathogenic mechanisms to treatment. Brain. 2017;140(3):527-46. https://doi.org/10.1093/brain/ aww258

PMid:27794524

5. Rocca MA, Amato MP, De Stefano N, Enzinger C, Geurts JJ, Penner IK, et al. Clinical and imaging assessment of cognitive dysfunction in multiple sclerosis. Lancet Neurol. 2015;14(3):30217. https://doi.org/10.1016/s1474-4422(14)70250-9 PMid:25662900

6. Kusumadewi W, Imran D, Witjaksono F, Pakasi TA, Rusmana AI, Pangeran D, et al. Low Vitamin D-25(OH) level in Indonesian multiple sclerosis and neuromyelitis optic patients. Mult Scler Relat Disord. 2018;25:329-33. https://doi.org/10.1016/j. msard.2018.08.030

PMid:30195201

7. Yueniwati Y, Wangsadjaja C, Yulidani II, Rianawati SS, A Rasyid $\mathrm{H}$. The role of brain magnetic resonance imaging (MRI) as an early detector of cognitive impairment. J Neurosci Rural Pract. 2018;9(3):350-3. https://doi.org/10.4103/jnrp. jnrp_542_17

PMid:30069090 
8. Neema M, Ceccarelli A, Jackson JS, Bakshi R. Magnetic resonance imaging in multiple sclerosis. In: Multiple Sclerosis: Diagnosis and Therapy. United States: Wiley; 2012. https://doi. org/10.1002/9781119963714.ch6

9. Brownlee WJ, Hardy TA, Fazekas F, Miller DH. Diagnosis of multiple sclerosis: Progress and challenges. Lancet. 2017;389(10076):1336-46.

PMid:27889190
10. Thompson AJ, Banwell BL, Barkhof F, Carroll WM, Coetzee T, Comi G, et al. Diagnosis of multiple sclerosis: 2017 revisions of the McDonald criteria. Lancet Neurol. 2018;17(2):162-73.

PMid:29275977

11. Marvanova Marketa. Multiple Sclerosis and Its Symptoms 2013. Available from: https://www.researchgate.net/ publication/305681128_Multiple_Sclerosis_and_Its Symptoms. [Last accessed on 2020 Oct 05] 